\section{Tratamiento y prevención de la mucositis oral asociada al tratamiento del cáncer}

\author{
GONZALO RUIZ-ESQUIDE ${ }^{1, \mathrm{a}}$, BRUNO NERVI ${ }^{2}$, \\ ALEX VARGAS ${ }^{3}$, ALBERTO MAÍZ $^{1}$
}

\section{Treatment and prevention of cancer treatment related oral mucositis}

One of the most common and troublesome complications of modern intensive anticancer treatments is oral mucositis. The purpose of this review is to summarize current evidence and clinical guidelines regarding its prevention and therapy. The use of keratinocyte growth factor-1, supplementary glutamine and other recently developed treatment modalities are discussed. The injury of the oral mucosa caused by antineoplastic agents promotes the local expression of multiple pro-inflammatory and pro-apoptotic molecules and eventually leads to the development of ulcers. Such lesions predispose patients to several infectious and nutritional complications. Also, they lead to modification of treatment schedules, potentially affecting overall prognosis. Local cryotherapy with ice chips and phototherapy with low energy laser may be useful as preventive measures. Mouthwashes with allopurinol and phototherapy with low energy laser can be used as treatment. In radiotherapy, special radiation administration techniques should be used to minimize mucosal injury. Pain control should always be optimized, with the use of patient controlled analgesia and topical use of morphine. Supplemental glutamine should not be used outside of research protocols. Lastly, thorough attention should be paid to general care and hygiene measures.

(Rev Med Chile 2011; 139: 373-381).

Key words: Antineoplastic protocols; Cryotherapy; Mucositis.

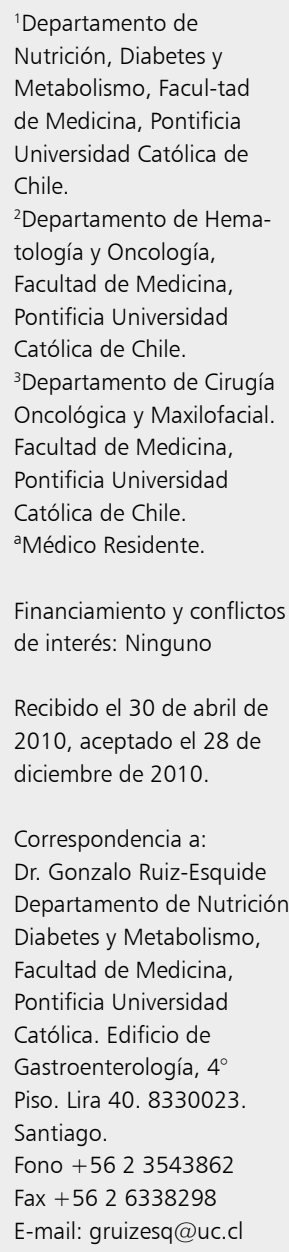

L a mucositis oral asociada al tratamiento del cáncer es una complicación frecuente tanto de la radioterapia como de la quimioterapia. Puede, incluso, llegar a ser el efecto adverso de la quimioterapia intensiva que más incomoda a los pacientes. ${ }^{1}$ Los objetivos de esta revisión son exponer la evidencia actualizada sobre medidas efectivas en la prevención y el tratamiento de la mucositis oral, específicamente la relativa al uso de glutamina suplementaria, de factor de crecimiento de keratinocitos, otras terapias desarrolladas recientemente y revisar las recomendaciones internacionales más importantes al respecto.

En general, el cáncer se caracteriza por una desregulación de la proliferación celular, con un aumento de la proliferación y disminucion de la apoptosis. Los tratamientos antineoplásicos convencionales se dirigen, por tanto, a bloquear la proliferación. Sin embargo, la acción de la quimioterapia y la radioterapia no es específica, por lo que en el contexto de tratamientos antineoplásicos es frecuente observar daños a tejidos que en condiciones fisiológicas tienen altas tazas de replicación celular. Entre ellos destaca la toxicidad en las mucosas oral y gastrointestinal. Luego de la injuria química o física a la mucosa oral derivada de los tratamientos anti neoplásicos comienzan a expresarse diversas moléculas proinflamatorias y proapoptóticas en las capas basales del epitelio $y$ en el estroma subepitelial. Las señales inflamatorias iniciales se amplifican por la activación local del factor de trascripción nuclear kB (NF- 
$\kappa \beta)$ y el aumento consecuente de la expresión de citoquinas proinflamatorias como el factor de necrosis tumoral $\alpha$ (TNF- $\alpha$ ). Esto promueve la transcripción de genes que codifican kinasas activadas por mitógenos (MAPK), cicloxigenasa2 (COX2) y otras moléculas de señalización que finalmente activan enzimas proteolíticas, como las metaloprotenasas, que son las efectores finales del proceso que termina por producir una úlcera (Figura 1). Esta, a su vez, se coloniza con bacterias, aumentando la expresión local de citoquinas e in- tensificando el proceso inflamatorio. Finalmente, apoyado por señales replicativas y diferenciadoras del estroma, el epitelio comienza a repararse y la úlcera cierra (Figura 2) ${ }^{2}$. Este proceso comienza habitualmente 3 a 7 días después de iniciada la quimioterapia, $o$ al alcanzar dosis acumuladas de 30 Gy de radioterapia y dura aproximadamente 2 a 3 semanas $^{2,3}$.

La clasificación de gravedad más utilizada en investigación ${ }^{4}$ es la propuesta por la Organización Mundial de la Salud (OMS) con una escala que

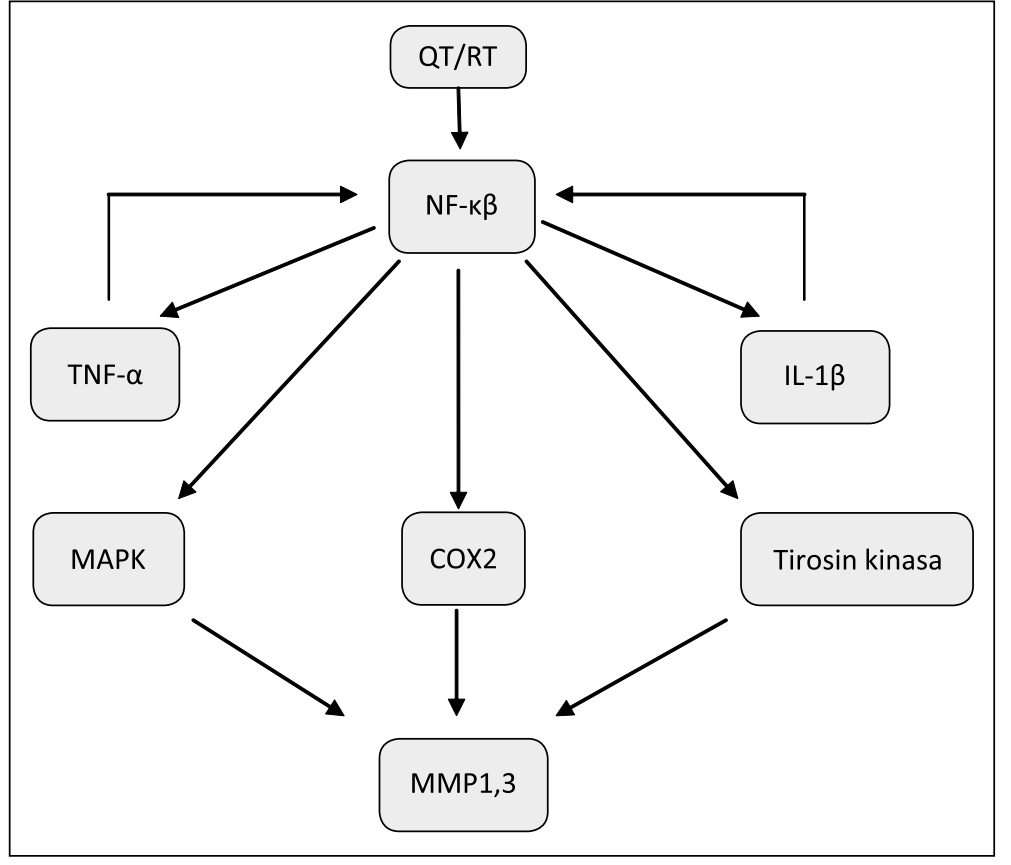

Figura 1. Amplificación de señales durante la mucositis. La quimio y radioterapia activan al factor de transcripción nuclear kB (NF- $\kappa \beta)$ en células epiteliales, endoteliales, mesenquimáticas y macrófagos, aumentando la expresión de citoquinas proinflamatorias, como el factor de necrosis tumoral $\alpha$ (TNF- $\alpha$ ) y la interleuquina $1 \beta$ (IL-1 $\beta$ ). Estas amplifican la señal primaria y activan al $\mathrm{NF}-\kappa \beta$ en otras células, lo que promueve la transcripción de genes que codifican kinasa activada por mitógeno (MAPK), ciclooxigenasa2 (COX2) y móleculas de señalización tirosin kinasa. Estas vías convergen en la activación de metaloproteinasas de matriz 1 y 3 (MMP1,3) en las células de del epitelio y la lámina propria, las efectoras finales de la injuria. Modificado, con permiso, de Macmillan Publishers Ltd, referecia².

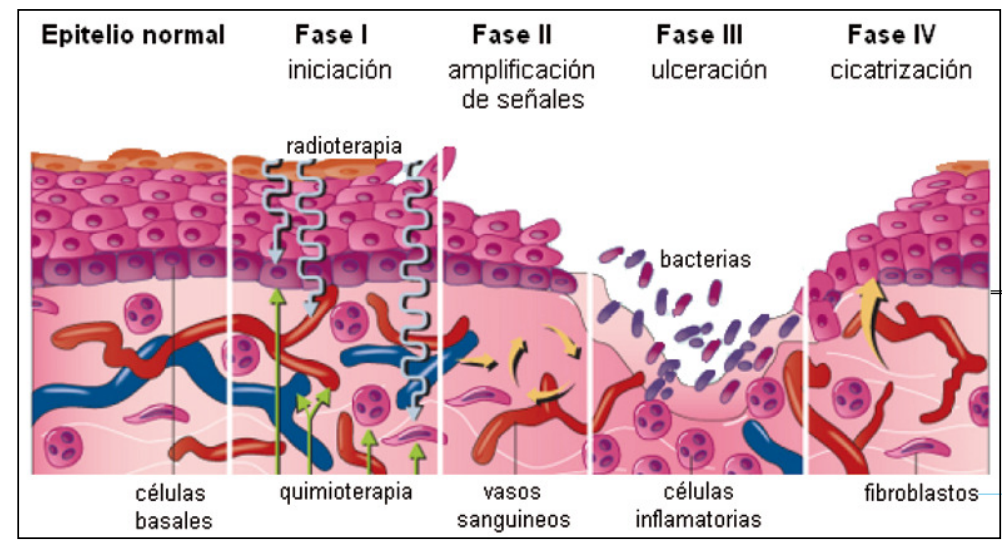

Figura 2. Fases en el desarrollo de la mucositis oral. Modificada con permiso de Elsevier Oncology, de referencia ${ }^{14}$. 
combina el aspecto clínico de la mucosa con la capacidad de ingerir alimentos ${ }^{5}$. En tanto que en clínica es más utilizada la escala que forma parte del manual "Common Toxicity Criteria for Adverse Events" del Instituto Nacional del Cáncer de los Estados Unidos de Norteamérica. Esta clasificación enfatiza los síntomas del paciente, la capacidad de mantener la ingesta oral y la necesidad de implemetar terapias $^{6}$ (Tabla 1 ).

La mucositis oral y gastrointestinal presenta un espectro sintomático y clínico complejo. Coexisten alteraciones hidroelectrolíticas secundarias a la diarrea; dolor, debilitamiento de la inmunidad de barrera y hemorragias secundarias a la ulceración de la mucosa y desnutrición por ingesta disminuida y malabsorción ${ }^{7}$. También condicionan frecuentemente atrasos, suspensiones o reducciones en la intensidad de los tratamientos con una disminución en la posibilidad de control de su enfermedad y un potencial aumento en la morbimortalidad $^{2,8}$. El diagnóstico diferencial incluye infecciones orofaríngeas virales, bacterianas y micóticas, y la enfermedad de injerto contra huésped (EIH), entidades respecto de las cuales se debe mantener un alto grado de sospecha y que requieren tratamiento específico y oportuno 9 . Por otro lado, la injuria a la mucosa oral y gastrointestinal predispone tener complicaciones infecciosas locales y sistémicas y que ellas tengan peor pronóstico y requieran un manejo más complejo ${ }^{10,11}$.

Dependiendo del sitio del tumor y del tratamiento usado la mucositis oral puede llegar a afectar prácticamente a todos los pacientes. Habitualmente hay correlación entre la intensidad del tratamiento y el daño observado, aunque la susceptibilidad individual a esta complicación es variable. Por ejemplo, Wardley y cols ${ }^{12}$ encontraron que en una serie de 429 pacientes sometidos a tratamientos de alta intensidad y trasplante de médula ósea, $100 \%$ experimentó mucositis oral como efecto adverso de la terapia de condicionamiento y en $67 \%$ de los casos, esta fue severa (grados 3 ó 4 de la OMS). La incidencia máxima se produjo hasta 18 días después de iniciada la quimioterapia y la mitad de los pacientes recibieron analgesia con opioides por un promedio de 6 días. En un metaanálisis de datos obtenidos de estudios aleatorios, Trotti ${ }^{4}$ encontró que $80 \%$ de los pacientes sometidos a radioquimioterapia por carcinomas de cabeza y cuello presentaron mucositis, con $57 \%$ de los pacientes en radioterapia con fraccionamientos no convencionales sufriendo mucositis grados 3 ó 4 . Sólo un tercio de los estudios reportó baja de peso, que llegó a 6 a $12 \%$ del peso inicial, planteando la interrogante de hasta qué punto el aspecto nutricional de esta complicación frecuentemente no es considerado. En tanto, en otras formas de tratamiento con radio o quimioterapia menos agresivas hacia la mucosa la incidencia de mucositis oral varía entre $1 \mathrm{y}$ $13 \% .{ }^{13}$ En la mucositis asociada a quimioterapia, la magnitud del daño depende de las drogas utilizadas, de la duración del tratamiento y la dosis. La administración prolongada o repetitiva de bajas dosis de citotóxicos se asocian a un mayor riesgo de mucositis, comparado con la infusión en bolos. Las drogas que afectan la síntesis de $\mathrm{ADN}$ tienen la mayor incidencia de mucositis, como por ejemplo los antimetabolitos (metotrexato, 5-fluoracilo) y los análogos de purinas (citarabina) con incidencias cercanas a 40-60\%. Otros antineoplásicos asociados con frecuencia a mucositis son el etopósido,

Tabla 1. Escalas de severidad de la mucositis oral comúnmente utilizadas

\begin{tabular}{|c|c|c|c|}
\hline \multicolumn{2}{|r|}{ OMS } & \multicolumn{2}{|r|}{$\mathbf{N C l}$} \\
\hline 0 & Sin evidencias subjetivas u objetivas de mucositis & 1 & $\begin{array}{l}\text { Asintomático o síntomas leves. Sin indicación de } \\
\text { intervenir }\end{array}$ \\
\hline 1 & Dolor oral con o sin eritema, sin úlceras & 2 & $\begin{array}{l}\text { Dolor moderado, no interfiere con la ingesta } \\
\text { oral. Se debe modificar la dieta }\end{array}$ \\
\hline 2 & Eritema y ulceración: puede tragar sólidos & 3 & Dolor severo, interfiere con la ingesta oral \\
\hline 3 & Eritema y ulceración: no puede tragar sólidos & 4 & $\begin{array}{l}\text { Consecuencias potencialmente letales, se requie- } \\
\text { re intervención urgente }\end{array}$ \\
\hline 4 & Eritema y ulceración: no puede alimentarse & 5 & Muerte \\
\hline
\end{tabular}

OMS = Organización Mundial de la Salud ${ }^{5} . \mathrm{NCl}=$ National Cancer Institute, Common Toxicity Criteria for Adverse Events ${ }^{6}$. 
ciclofosfamida, doxorrubicina, daunorrubicina, docetaxel y paclitaxel. El metotrexato y el etopósido se secretan a la saliva, con lo que su toxicidad oral aumenta ${ }^{3,4,14,15}$.

\section{Evidencia en prevención y tratamiento}

Worthington y cols $^{16}$ publicaron en la base Cochrane una revisión sistemática de trabajos aleatorizados sobre prevención de mucositis. La metodología del estudio es sólida, con una buena pregunta, búsqueda amplia y exhaustiva, revisión y calificación de los trabajos encontrados por dos investigadores en forma independiente y reproducible $^{17}$. Encontraron 89 trabajos que evaluaban 33 intervenciones diferentes en 7.523 pacientes. $\mathrm{La}$ intervención que acumula la mayor cantidad de publicaciones es el uso de amifostina parenteral (Ethyol ${ }^{\circledR}$ ), un amino thiol capturador de radicales libres, con 11 trabajos que acumulan 845 pacientes. Sin embargo, el efecto de la amifostina fue discreto, con un riesgo relativo de presentar mucositis leve o moderada en el grupo tratado de 0,95 (IC $95 \%=0,92-0,98)$ y 0.88 (IC $95 \%=$ $0,80-0,98)$ respectivamente. Otras medidas con resultados positivos fueron: enzimas hidrolíticas por vía oral, crioterapia oral con hielo y diferentes combinaciones de hierbas tradicionales chinas.

Los mismos autores llevaron a cabo una segunda revisión sistemática de estudios de tratamiento ${ }^{18}$, en el que detectaron 26 trabajos susceptibles de analizar con 1.353 pacientes. Quince de estos eran propiamente sobre tratamiento y 12 sobre analgesia. Encontraron que 4 intervenciones tenían un estudio con resultados positivos, todos con riesgo moderado o alto de sesgo: 1) colutorios con alopurinol;2) inyecciones subcutánes de factor estimulador de colonias de granulocítos-macrófagos (GM-CSF); 3) inyecciones intramusculares de extracto de placenta humana y 4) inmunoglobulinas polivalentes intramusculares. De ellos sólo el alopurinol fue capaz de erradicar la mucositis (300 mg disueltos en agua o solución salina isotónica o un preparado comercial al efecto). Los demás la disminuían parcialmente. Los trabajos de analgesia demuestran que la técnica de analgesia controlada por el paciente (ACP) logra similares niveles de alivio que la infusión contínua de opioides, pero con menores dosis totales. No se incluyeron en este trabajo algunas investigaciones recientes sobre uso tópico de morfina que tienen resultados promisorios (por ejemplo, colutorios de $30 \mathrm{mg}$ en $15 \mathrm{cc}$ de agua, 6 veces al día) ${ }^{19-21}$.

En ambas revisiones se recalca que la metodología, la calidad, o ambos, de los reportes de los trabajos originales son en general deficientes, por lo que es necesario contar con estudios clínicos bien diseñados y de mayor tamaño que permitan sacar conclusiones más firmes, preocupación que otros expertos han manifestado independientemente ${ }^{22}$.

Recientemente se ha reunido una considerable casuística sobre el uso de fototerapia con láser de baja intensidad aplicada a la mucosa oral tanto en prevención como en tratamiento de la mucositis asociada a quimio y/o radioterapia (Figura 3 ). Este tipo de tratamiento acumula más de 40 años de experiencia en el manejo de lesiones dolorosas y procesos inflamatorios locales ${ }^{23,24}$. La bioestimulación con bajas energías (entre 120 y 240 mJ/ $\mathrm{cm}^{2}$ ) ha demostrado diversos beneficios en los procesos reparativos tisulares ${ }^{25-29}$. Hay aproximadamente diez estudios aleatorizados sobre el uso del láser de baja intensidad aplicada a la mucosa oral, tanto en prevención como en tratamiento de mucositis asociada a quimio y/o radioterapia. $\mathrm{La}$ mayor parte de ellos fueron publicados después de la última actualización de las revisiones mencionadas en los párrafos precedentes. Los reportes anteriores no habían sido incluidos en ellas, en su mayor parte, porque los resultados no estaban expresados de una forma susceptible de incluir en un metaanálsis, y no por metodología defectuosa. Cuatro de ellos fueron realizados en adultos y destacan por buena manera de agrupar en forma aleatoria $^{30}$, una muy buena mantención del ciego, tanto de los pacientes como de los adjudicadores de eventos ${ }^{31}$, una muy buena metodología en

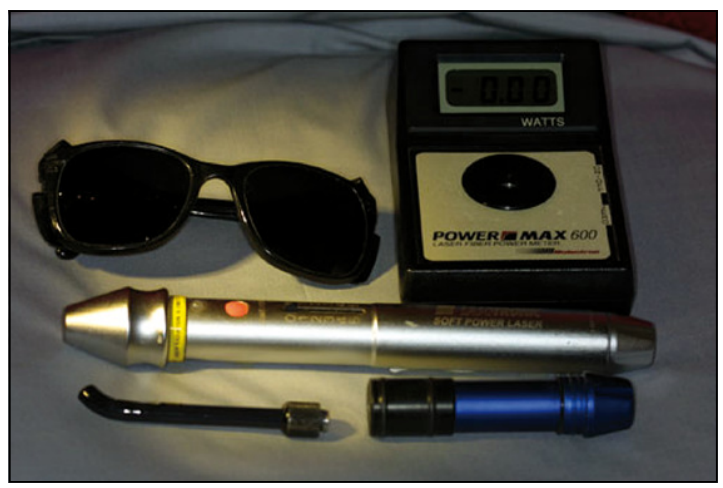

Figura 3. Equipo de láser de baja intensidad. 
general ${ }^{32}$, y haberse realizado en poblaciones de alto riesgo. Los primeros dos trabajos se realizaron en pacientes sometidos a quimio y/o radioterapia como tratamiento de cánceres de cabeza y cuello. Bensadoun y cols estudiaron 30 casos $^{33}$. Encontraron una incidencia de mucositis grado 3 de la OMS de $35 \%$ en el grupo control y de $7 \%$ en el tratado, con un número necesario a tratar (NNT) de 4 para prevenir una mucositis grado 3 de la OMS. Arun Maiya y cols estudiaron a 25 pacientes. ${ }^{34}$ Todos los del grupo control tuvieron mucositis grado 3 ó 4, en tanto que los tratados sólo tuvieron grado 1 ó 2, lo que da un NNT de 1 para prevenir una mucositis severa. Antunes y cols estudiaron el efecto de aplicaciones diarias de láser durante la fase de condicionamiento pretrasplante de médula ósea hasta la recuparación del conteo de neutrófilos ${ }^{35}$. Uno de 19 pacientes en el grupo tratado tuvo mucositis grado 3 , en tanto que 13 de 19 del grupo control la desarrollaron en grados 3 ó 4, lo que da una relación de riesgo de 0,07 (rango, $0,11-0,53 ; \mathrm{p}<, 001)$ y un NNT de 1 para prevenir una mucositis severa. Por último, Genot-Klastersky y cols reportan el resultado de un estudio de dos ramas: prevención en pacientes que habían desarrollado mucositis severa en ciclos previos de tratamiento para diferentes tumores y tratamiento en pacientes con mucositis activa ${ }^{36}$. Treinta y seis pacientes con mucositis activa grados 1 ó 2 de la OMS fueron asignados al azar a fototerapia o a un procedimiento simulado. Tres de 18 pacientes en el grupo de estudio y 16 de 18 en el grupo control progresaron al grado 3 , lo que representa un riesgo relativo de progresar a mucositis severa de 0,19 (Intervalo de Confianza 95\% $=0,07-0,53$ ) y un NNT de 1 (cálculos realizados por nosotros con los datos originales). En todos los reportes destaca una excelente tolerabilidad del procedimiento, sin aumento de infecciones y prácticamente sin efectos adversos atribuibles al uso del láser.

\section{Guías clínicas}

Tres guías merecen un comentario en el presente trabajo por ser las más citadas en el tema ${ }^{8,22,37}$. Al revisarlas destacan algunos elementos (Tabla 2). En primer lugar, los protocolos de higiene y cuidados generales orales que se promueven no tienen una base sólida con evidencias científicamente demostradas, pero el consenso es que son relevantes. Se hace especial referencia a la importancia de una evaluación y tratamiento dental meticuloso antes de comenzar las terapias antineoplásicas.

La crioterapia se recomienda en colutorios de 20-30 minutos previo a tratamientos con bolos de 5-FU, edatrexato y a uso de dosis altas

Tabla 2. Resumen de medidas con recomendaciones positivas en guías clínicas de prevención y tratamiento de mucositis

\begin{tabular}{|c|c|c|c|c|}
\hline & & MASCC/ISOO' & $\mathrm{ASCO}^{22}$ & ESMO $^{37}$ \\
\hline & & $\begin{array}{l}\text { Higiene, cuidados generales y } \\
\text { analgesia con ACP }\end{array}$ & - & $\begin{array}{l}\text { Higiene, cuidados generales y } \\
\text { analgesia con ACP }\end{array}$ \\
\hline \multirow[t]{3}{*}{ Radioterapia } & Prevención & $\begin{array}{l}\text { Bloqueos de línea media y } \\
\text { modelamiento tridimensional }\end{array}$ & - & $\begin{array}{l}\text { Bloqueos de línea media y } \\
\text { modelamiento tridimensional }\end{array}$ \\
\hline & & Benzamidina & - & - \\
\hline & Tratamiento & - & - & - \\
\hline \multirow[t]{2}{*}{ Quimioterapia convencional } & Prevención & Crioterapia & - & $\begin{array}{l}\text { Crioterapia } \\
\text { KGF-1 }\end{array}$ \\
\hline & Tratamiento & - & - & - \\
\hline $\begin{array}{l}\text { Quimioterapia en dosis altas } \\
\text { con o sin ICT más TCTH }\end{array}$ & Prevención & $\begin{array}{l}\text { KGF-1 } \\
\text { Crioterapia } \\
\text { TLBE }\end{array}$ & KGF-1 & $\begin{array}{l}\text { KGF-1 } \\
\text { Crioterapia } \\
\text { TLBE }\end{array}$ \\
\hline
\end{tabular}

MASCC/ISOO = Multinational Association of Supportive Care in Cancer and the International Society for Oral Oncology. $\mathrm{ASCO}=$ American Society of Clinical Oncology. ESMO = European Society of Clinical Oncology. ACP = Analgesia Controlada por el Paciente. - = no hay recomendaciones específicas. ICT = Irradiación Corporal Total. TCTH = Trasplante de Células Troncales Hematopoyéticas. KGF-1 = Factor de Crecimiento de Keratinocitos-1 (palifermin). TLBE = Terapia con Láser de Baja Energía. 
de melphalan. La referencia en que se apoya la recomendación del uso de factor de crecimiento de keratinocitos como profilaxis en casos de acondicionamiento pretrasplante de médula ${ }^{38}$ está considerada en el metaanálisis de Worthington ${ }^{16}$, quienes agregaron sus resultados con otros dos estudios de resultados negativos ${ }^{39,40}$, razón por la que no fue mencionada en sus resultados como una intervención de efectos positivos. Las guías de la ASCO (American Society of Clinical Oncology) recomiendan la amifostina sólo para la prevención de la xerostomía asociada a la radioterapia aplicada a la cavidad oral, no para prevenir la mucositis. Por último, las terapias con láser de baja energía son reseñadas como promisorias, pero complicadas por el costo de los equipos y la necesidad de contar con personal especializado.

\section{Glutamina suplementaria}

La glutamina es uno de los aminoácidos más abundantes en el cuerpo humano, donde cumple un sinnúmero de funciones consideradas benéficas $^{41}$, especialmente derivadas de su capacidad de transportar un segundo grupo amino en su molécula, de su rol como fuente energética preferente de las células intestinales e inmunes y de su relación a la protección contra el estrés oxidativo por vía de la generación de glutatión $\mathrm{n}^{42-44}$. Puede ser sintetizada a nivel hepático y muscular en grandes cantidades, pero en enfermedades graves y en el cáncer se considera un aminoácido condicionalmente esencial, pues el organismo llega a depletarse profundamente de sus reservas, afectando la función inmune y potencialmente la barrera intestinal $^{42,45}$. Se ha usado en prevención o tratamiento de mucositis por vía oral, como colutorios que se tragan o por vía parenteral. Aunque hay estudios con resultados alentadores y recomendaciones de expertos $^{46}$, la controversia respecto de su utilidad y seguridad continúa ${ }^{42,47,48}$.

Recientemente fue publicado un metaanálisis respecto del uso glutamina suplementaria en quimioterapia no asociada a trasplante de médula osea ${ }^{49}$. Este trabajo no presenta las fortalezas metodológicas mencionadas más arriba: la búsqueda se describe escuetamente como "una breve búsqueda en Pubmed", no hay revisión explícita ni independiente de la calidad de los trabajos originales y uno de ellos fue eliminado del análisis por deficiencias metodológicas que no están descritas.
Sin embargo, una búsqueda realizada por nosotros no ha identificado otros trabajos relevantes que los que menciona el autor. El autor encontró 6 estudios originales (con 591 pacientes) aleatorizados a glutamina oral o placebo y 3 (con 73 pacientes) asignados a glutamina parenteral o placebo. Los esquemas de tratamiento en los trabajos originales incluyen antraciclinas, 5-FU, radioquimioterapia, radioterapia y trabajos con esquemas variados. El riesgo relativo de desarrollar mucositis de cualquier grado con glutamina profiláctica fue 1,40 (IC $95 \%=0,96-2,05)$ y el riesgo de desarrollar mucositis severa fue 1,12 (IC $95 \%=0,68-1,84)$. La revisión sistemática de Worthington y cols, sobre prevención de la mucositis, identificó 6 trabajos que no demuestran resultados positivos al agregar los resultados ${ }^{16}$.

Un segundo metaanálisis sobre el uso de glutamina suplementaria se refiere específicamente a su uso en pacientes sometidos a trasplante de células hematopoyéticas ${ }^{50}$. Este estudio sí cuenta con una gran fortaleza metodológica y sus resultados tienen por tanto un bajo riesgo de sesgo. En él se incluyen 17 trabajos con uso oral o endovenoso de glutamina, los cuales tienen, en general, características metodológicas relativamente pobres. Hubo una tendencia a tener menor severidad promedio de la mucositis en el grupo asignado a glutamina, fundamentalmente dada por los pacientes que la recibieron por vía oral. Esta diferencia alcanzó la significación estadística, pero con una magnitud clínica muy discreta (Diferencia media estándar en puntajes de severidad para glutamina vs control $=-0,24$, IC $95 \%=-0,42$ a $-0,05$ y 1,95 días menos de requerimientos de opioides en promedio). No hubo diferencias en la duración de la mucositis, ni en el riesgo de sufrir mucositis severa. El uso de glutamina no influyó en la mortalidad total a 100 días del trasplante $(\mathrm{RR}=0,88$, IC 95\% $=0,60$ a 1,27). En el caso de las infecciones clínicas, al considerar todos los trabajos hubo una reducción de riesgo estadísticamente significativa $(R R=0,77$, IC $95 \%=0,60$ a 0,98 ), que no se mantuvo al repetir el análisis sólo con los trabajos de mejor calidad. Sí se encontró que el uso de glutamina endovenosa (dos estudios, 70 pacientes) se asociaba a aumento de riesgo de sufrir recaídas de la enfermedad de base a seis meses ( $R R=2,91$, IC $95 \%=1,34$ a 6,29) (Tabla 3). La combinación de resultados en un solo estimador puntual se dificulta en este metaanálisis porque los diferentes trabajos originales reporta- 
Tabla 3. Metaanálisis de efectos de la glutamina suplementaria en la prevención de mucositis en pacientes sometidos a trasplante de células hematopoyéticas

\begin{tabular}{|lll|}
\hline Comparación & $\begin{array}{c}\text { N estudios, n de } \\
\text { pacientes }\end{array}$ & Resultado \\
\hline $\begin{array}{l}\text { Puntaje promedio de mucositis, glutamina oral } \\
\text { Puntaje promedio de mucositis, glutamina ev }\end{array}$ & $\mathrm{N}=4, \mathrm{n}=329$ & $\mathrm{DME}=-0,38\left(95 \% \mathrm{Cl}-0,59 \mathrm{a}-0,16, \mathrm{I}^{2}=27 \%\right)$ \\
\hline $\begin{array}{l}\text { Puntaje promedio de mucositis, general } \\
\text { Días de mucositis, oral }\end{array}$ & $\mathrm{N}=4, \mathrm{n}=131$ & $\mathrm{DME}=-0,24\left(95 \% \mathrm{Cl}-0,42\right.$ a $\left.-0,05, \mathrm{I}^{2}=48 \%\right)$ \\
Días de mucositis, general & $\mathrm{DME}=-0,26\left(95 \% \mathrm{Cl}-1,02\right.$ a $\left.0,50, \mathrm{I}^{2}=59 \%\right)$ \\
Mucositis severa, oral & $\mathrm{N}=2, \mathrm{n}=52$ & $\mathrm{DME}=-0,31\left(95 \% \mathrm{Cl}-0,82\right.$ a $\left.0,20, \mathrm{I}^{2}=45 \%\right)$ \\
Puntaje máximo de mucositis, general & $\mathrm{N}=4, \mathrm{n}=352$ & $\mathrm{DM}=0,81\left(95 \% \mathrm{Cl} 0,58\right.$ a $\left.1.14, \mathrm{I}^{2}=46 \%\right)$ \\
\hline $\begin{array}{l}\text { Días de opioides, oral } \\
\text { Días de opioides, general }\end{array}$ & $\mathrm{DM}=-0,09\left(95 \% \mathrm{Cl}-0,61\right.$ a $\left.0,43, \mathrm{I}^{2}=67 \%\right)$ \\
\hline
\end{tabular}

$\mathrm{DME}=$ diferencia media estándar; $\mathrm{RR}=$ riesgo relativo; $\mathrm{DM}=$ diferencia media; $\mathrm{IC}=$ intervalo de confianza. Datos de referencia ${ }^{50}$.

ron la incidencia y severidad de la mucositis de distinto modo, presentan amplias variaciones en las características de los pacientes estudiados y en la dosificación de la glutamina (aproximadamente 1,02 a $7,31 \mathrm{~g} / \mathrm{k} / \mathrm{día}$ ). Más aun, los niveles de heterogeneidad de los resultados (con valores de $\mathrm{I}^{2}$ de 45 a $84 \%$ ) limitan la confianza con que se puede llegar a cualquier conclusión en base a estos datos ${ }^{51,52}$.

Ninguna de las guías clínicas consultadas recomienda el uso de glutamina suplementaria ${ }^{8,22,37}$, e incluso hacen presente ciertas preocupaciones respecto de la posibilidad de que su uso aumente la recidiva de algunos tumores ${ }^{8}$.

\section{Conclusiones}

La mucositis oral es una complicación frecuente de las terapias antineoplásicas, cuya presentación clínica va desde dolor local leve hasta ulceración severa, hemorragia y afagia. Se requieren estudios clínicos bien llevados, idealmente de acuerdo a las recomendaciones CONSORT ${ }^{53}$, que nos permitan avanzar en el conocimiento de medidas profilácticas y terapéuticas efectivas. Dada la naturaleza subjetiva de las manifestaciones de la mucositis un aspecto especialmente importante a la hora de juzgar el riesgo de sesgo de un trabajo es que se haya cuidado bien la mantención del ciego de los adjudicadores de resultados.

Considerando la evidencia y las recomendaciones expuestas, las intervenciones que nos parecen más recomendables son: en prevención, la crioterapia con hielo local y la fototerapia con láser de baja energía; y en terapia los colutorios con alopurinol y la fototerapia con láser. En radioterapia hay que considerar siempre la mucosa oral como un órgano crítico para restringir, en la medida de lo posible, la dosis de radiación que ella recibe. La analgesia debería optimizarse en todos los casos, probablemente con opioides administrados con ACP por vía sistémica o colutorios de morfina. No debería usarse, por ahora, glutamina suplementaria como tratamiento ni como profilaxis fuera de protocolos de investigación. Por último, no nos parece que deban abandonarse las medidas básicas de higiene y cuidados generales que el paciente requiera sólo porque no cuentan con ensayos aleatorizados que las respalden.

\section{Referencias}

1. Bellm LA, Epstein JB, Rose-Ped A, Martin P, Fuchs HJ. Patient reports of complications of bone marrow transplantation. Support Care Cancer 2000; 8: 33-9.

2. Sonis ST. The pathobiology of mucositis. Nat Rev Cancer 2004; 4: 277-84.

3. Sideras K, Loprinzi C, Foote R. Oral complications. En: Abeloff M, Armitage J, Niederhuber J, Kastan M, McKenna WG, editores. Clinical Oncology. 4th ed. Philadelphia: Churchill Livingstone, an imprint of Elsevier Inc.; 2008. p. 609-24.

4. Trotti A, Bellm LA, Epstein JB, Frame D, Fuchs HJ, Gwede CK, et al. Mucositis incidence, severity and associated outcomes in patients with head and neck cancer 
receiving radiotherapy with or without chemotherapy: A systematic literature review. Radiother Oncol 2003; 66: 253-62.

5. World Health Organization. WHO handbook for reporting results of cancer treatment. Geneva: World Health Organization: WHO Offset Publication; 1979.

6. Common terminology criteria for adverse events (CTCAE) v4.0 [Página en Internet]. National Cancer Institute. 201014 de junio de 2010 [citada 25 de noviembre de 2010]. Disponible de: http://evs.nci.nih.gov/ftp1/ CTCAE/About.html.

7. Rosenthal DI. Consequences of mucositis-induced treatment breaks and dose reductions on head and neck cancer treatment outcomes. J Support Oncol 2007; 5: 23-31.

8. Keefe DM, Schubert MM, Elting LS, Sonis ST, Epstein JB, Raber-Durlacher JE, et al. Updated clinical practice guidelines for the prevention and treatment of mucositis. Cancer 2007; 109: 820-31.

9. Epstein JB. Mucositis in the cancer patient and immunosuppressed host. Infect Dis Clin North Am 2007; 21: 503, 22, vii.

10. Marti FM, Cullen MH, Roila F, ESMO Guidelines Working Group. Management of febrile neutropenia: ESMO clinical recommendations. Ann Oncol 2009; 20 Suppl 4: 166-9.

11. Hughes WT, Armstrong D, Bodey GP, Bow EJ, Brown AE, Calandra T, et al. 2002 guidelines for the use of antimicrobial agents in neutropenic patients with cancer. Clin Infect Dis 2002; 34: 730-51.

12. Wardley AM, Jayson GC, Swindell R, Morgenstern GR, Chang J, Bloor R, et al. Prospective evaluation of oral mucositis in patients receiving myeloablative conditioning regimens and haemopoietic progenitor rescue. $\mathrm{Br}$ J Haematol 2000; 110: 292-9.

13. Jones JA, Avritscher EB, Cooksley CD, Michelet M, Bekele BN, Elting LS. Epidemiology of treatment-associated mucosal injury after treatment with newer regimens for lymphoma, breast, lung, or colorectal cancer. Support Care Cancer 2006; 14: 505-15.

14. Peterson DE. New strategies for management of oral mucositis in cancer patients. J Support Oncol 2006; 4: 9-13.

15. Rubenstein EB, Peterson DE, Schubert M, Keefe D, McGuire D, Epstein J, et al. Clinical practice guidelines for the prevention and treatment of cancer therapy-induced oral and gastrointestinal mucositis. Cancer 2004; 100: 2026-46.

16. Worthington HV, Clarkson JE, Eden OB. Interventions for preventing oral mucositis for patients with cancer receiving treatment. Cochrane Database Syst Rev 2007;

\section{(4): CD000978.}

17. Letelier LM, Manriquez JJ, Rada G. [Systematic reviews and metaanalysis: Are the best evidence?] Rev Med Chile 2005; 133: 246-9.

18. Clarkson JE, Worthington HV, Eden OB. Interventions for treating oral mucositis for patients with cancer receiving treatment. Cochrane Database Syst Rev 2007; (2): CD001973.

19. Cerchietti L. Morphine mouthwashes for painful mucositis. Support Care Cancer. 2007; 15: 115-6; author reply 117.

20. LeBon B, Zeppetella G, Higginson IJ. Effectiveness of topical administration of opioids in palliative care: A systematic review. J Pain Symptom Manage 2009; 37: 913-7.

21. Cerchietti LC, Navigante AH, Bonomi MR, Zaderajko MA, Menendez PR, Pogany CE, et al. Effect of topical morphine for mucositis-associated pain following concomitant chemoradiotherapy for head and neck carcinoma. Cancer 2002; 95: 2230-6.

22. Hensley ML, Hagerty KL, Kewalramani T, Green DM, Meropol NJ, Wasserman TH, et al. American society of clinical oncology 2008 clinical practice guideline update: Use of chemotherapy and radiation therapy protectants. J Clin Oncol 2009; 27: 127-45.

23. Mester E, Nagylucskay S, Doklen A, Tisza S. Laser stimulation of wound healing. Acta Chir Acad Sci Hung 1976; 17: 49-55.

24. Enwemeka CS. Therapeutic light. Rehab Manag 2004; 17: 20,5, 56-7.

25. Hrnjak M, Kuljic-Kapulica N, Budisin A, Giser A. Stimulatory effect of low-power density he-ne laser radiation on human fibroblasts in vitro. Vojnosanit Pregl 1995; 52: $539-46$.

26. Agaiby AD, Ghali LR, Wilson R, Dyson M. Laser modulation of angiogenic factor production by T-lymphocytes. Lasers Surg Med 2000; 26: 357-63.

27. Ghali L, Dyson M. The direct effect of light therapy on endothelial cell proliferation in vitro. EXS 1992; 61: 4114.

28. Hu WP, Wang JJ, Yu CL, Lan CC, Chen GS, Yu HS. Helium-neon laser irradiation stimulates cell proliferation through photostimulatory effects in mitochondria. J Invest Dermatol 2007; 127: 2048-57.

29. Tran TD, Inui K, Hoshiyama M, Lam K, Kakigi R. Conduction velocity of the spinothalamic tract following CO2 laser stimulation of C-fibers in humans. Pain 2002; 95: 125-31.

30. Penaloza B, Candia R. [Why is it essential to randomize a clinical trial on therapy?] Rev Med Chile 2004; 132: 1007-10. 
31. Letelier LM, Manriquez JJ, Claro JC. [Blinding for clinical trials. Does it matter?] Rev Med Chile 2004; 132: 1137-9.

32. Guyatt GH, Sackett DL, Cook DJ. Users' guides to the medical literature. II. how to use an article about therapy or prevention. A. are the results of the study valid? evidence-based medicine working group. JAMA 1993; 270: 2598-601.

33. Bensadoun RJ, Franquin JC, Ciais G, Darcourt V, Schubert MM, Viot M, et al. Low-energy $\mathrm{He} / \mathrm{Ne}$ laser in the prevention of radiation-induced mucositis. A multicenter phase III randomized study in patients with head and neck cancer. Support Care Cancer 1999; 7: 244-52.

34. Arun Maiya G, Sagar MS, Fernandes D. Effect of low level helium-neon (He-Ne) laser therapy in the prevention \& treatment of radiation induced mucositis in head \& neck cancer patients. Indian J Med Res 2006; 124: 399402.

35. Antunes HS, de Azevedo AM, da Silva Bouzas LF, Adao CA, Pinheiro CT, Mayhe R, et al. Low-power laser in the prevention of induced oral mucositis in bone marrow transplantation patients: A randomized trial. Blood 2007; 109: 2250-5.

36. Genot-Klastersky MT, Klastersky J, Awada F, Awada A, Crombez P, Martinez MD, et al. The use of low-energy laser (LEL) for the prevention of chemotherapy- and/or radiotherapy-induced oral mucositis in cancer patients: Results from two prospective studies. Support Care Cancer 2008; 16: 1381-7.

37. Peterson DE, Bensadoun R-, Roila F, On behalf of the ESMO Guidelines Working Group,. Management of oral and gastrointestinal mucositis: ESMO clinical recommendations. Ann Oncol 2009; 20: iv174-177.

38. Spielberger R, Stiff P, Bensinger W, Gentile T, Weisdorf $\mathrm{D}$, Kewalramani $\mathrm{T}$, et al. Palifermin for oral mucositis after intensive therapy for hematologic cancers. $N$ Engl J Med 2004; 351: 2590-8.

39. Freytes CO, Ratanatharathorn V, Taylor C, Abboud C, Chesser N, Restrepo A, et al. Phase I/II randomized trial evaluating the safety and clinical effects of repifermin administered to reduce mucositis in patients undergoing autologous hematopoietic stem cell transplantation. Clin Cancer Res 2004; 10: 8318-24.

40. Meropol NJ, Somer RA, Gutheil J, Pelley RJ, Modiano MR, Rowinsky EK, et al. Randomized phase I trial of recombinant human keratinocyte growth factor plus chemotherapy: Potential role as mucosal protectant. J Clin Oncol 2003; 21: 1452-8.

41. McClave SA, Martindale RG, Vanek VW, McCarthy M, Roberts P, Taylor B, et al. Guidelines for the provision and assessment of nutrition support therapy in the adult critically ill patient: Society of critical care medicine (SCCM) and american society for parenteral and enteral nutrition (A.S.P.E.N.). JPEN J Parenter Enteral Nutr 2009; 33: 277-316.

42. Savarese DM, Savy G, Vahdat L, Wischmeyer PE, Corey B. Prevention of chemotherapy and radiation toxicity with glutamine. Cancer Treat Rev 2003; 29: 501-13.

43. Tawa NE, Fisher JE. Metabolism in surgical patients. En: Townsend CM, Beauchamp DR, Evers M, Mattox KL, editores. Townsend: Sabiston Textbook of Surgery. 18th ed. Philadelphia, Pennsylvania: Elsevier Saunders; 2007. p. 143-87.

44. Guyton AC, Hall JE. Metabolism and temperature regulation. En: Textbook of Medical Physiology. 11th ed. Philadelphia, Pennsylvania: Elsevier, Saunders; 2005. p. 828-88.

45. Melis GC, ter Wengel N, Boelens PG, van Leeuwen PA. Glutamine: Recent developments in research on the clinical significance of glutamine. Curr Opin Clin Nutr Metab Care 2004; 7: 59-70.

46. August DA, Huhmann MB, the American Society for Parenteral and Enteral Nutrition (A.S.P.E.N.) Board of Directors. A.S.P.E.N. clinical guidelines: Nutrition support therapy during adult anticancer treatment and in hematopoietic cell transplantation. JPEN J Parenter Enteral Nutr 2009; 33: 472-500.

47. Murray SM, Pindoria S. Nutrition support for bone marrow transplant patients. Cochrane Database Syst Rev 2009; (1): CD002920.

48. Hardy ML. Dietary supplement use in cancer care: Help or harm. Hematol Oncol Clin North Am 2008; 22: 581, 617, vii.

49. Crowther M. Hot topics in parenteral nutrition. A review of the use of glutamine supplementation in the nutritional support of patients undergoing bone-marrow transplantation and traditional cancer therapy. Proc Nutr Soc 2009; 68: 269-73.

50. Crowther M, Avenell A, Culligan DJ. Systematic review and meta-analyses of studies of glutamine supplementation in haematopoietic stem cell transplantation. Bone Marrow Transplant 2009; 44: 413-25.

51. Higgins JP, Thompson SG, Deeks JJ, Altman DG. Measuring inconsistency in meta-analyses. BMJ 2003; 327: 557-60.

52. Higgins JP, Thompson SG. Quantifying heterogeneity in a meta-analysis. Stat Med 2002; 21: 1539-58.

53. Moher D, Schulz KF, Altman DG, CONSORT GROUP (Consolidated Standards of Reporting Trials). The CONSORT statement: Revised recommendations for improving the quality of reports of parallel-group randomized trials. Ann Intern Med 2001; 134: 657-62. 